\title{
Morphic Arrangement of High Flexibility and Aspect Ratio Wing
}

\author{
Wojciech SKARKA ${ }^{1}$ and Nikodem CIOMPERLIK ${ }^{2}$ \\ Silesian University of Technology
}

\begin{abstract}
Morphing of aerodynamic surfaces or conformal shape adaptation of aerodynamic surfaces can be used to control aircraft, utilized similarly as in nature, where insects and birds deform their wings to achieve a wide range of flight conditions. Morphing of wings has the potential to bring numerous advantages in flight performance in comparison to a rigid, conventional solution, that utilizes stiff aerodynamic surfaces. Reduction of parasitic drag due to the lack of gaps between the various moveable surfaces is one of them. Even so, a wing whose sections are able to deform independently or conform can better adapt to wide range of flight conditions than a rigid solution, or a solution based on conventional aerodynamic surfaces, such as flaps and ailerons. Additionally, the conformal shape adaptation or morphing of aerodynamic surfaces may lead to a potentially reduced weight and mechanical complexity, which may be achieved by utilizing wing deformations directly in the structure instead of connecting conventional actuation devices to the system. The aim of this paper is to propose a morphic arrangement of a high flexibility and high aspect ratio wing, that could be utilized in High Altitude Long Endurance aircraft, where the efficiency of the design is of utmost importance. A significant reduction of parasitic drag and reduction of weight is a promising basis for pursuing morphic and conformal shape adaptation designs. This paper qualitatively explores the space of morphic arrangements and conformal shape adaptation designs and utilizes inventive approaches to check and identify designs that may be promising. A wing design is proposed, that utilizes morphing of wing and conformal shape adaptation.
\end{abstract}

Keywords. morphing wing, high aspect ratio wing, high flexibility wing, aircraft control

\section{Introduction}

The idea of a morphing wing arrangement is a direct conflict with structural requirements, however as alternative methods for control of aerodynamic surfaces were developed, new materials and tools for optimization became available, such designs are becoming more popular and an active area of research. Morphing technologies are an area of interest due to the fact that they offer lowering of consumed power, environmental impact, emitted noise and cost of the aircraft, while providing a slightly better performance.

The main areas of improvements are stall characteristics, roll and stall control, as well as lift to drag ratio. Morphic wing arrangements can have a positive impact on

\footnotetext{
${ }^{1}$ Corresponding Author, Mail: Wojciech Skarka [wojciech.skarka@polsl.pl.

${ }^{2}$ Corresponding Author, Mail: Nikodem Ciomperlik [nikodemc@gmail.com.
} 
endurance and range characteristics. A wing of any shape can be optimized for a wide range of lift coefficient to generate the same amount of induced drag as an elliptic wing. Variable wing camber paired together with seamless skin is a promising solution for high noise generation during specific mission profile segments. It was also shown that such arrangements may improve aeroelastic efficiency and increase the critical flutter velocity [3].

Machines engineered by humans tend to be rigid, connected by different joins or interfaces, whereas morphing arrangements take inspiration from nature, where structures are able to adapt their shape in a very large range to maximize their performance and are often flexible or compliant, whilst still offering required strength [4].

The utilization of concepts found in nature that use flexibility in morphing designs is based on embracing elasticity, instead of avoiding it. Just like the mechanisms that are the base for this approach, it is essential to create a system that is both flexible and strong, able to withstand the dynamic forces and additionally simple, when number of parts are taken into account [6].

To achieve those contradictory quantities, mechanism sections need to simultaneously carry the loads as well as perform all kinematic functions. This approach leads to reduced mechanical complexity and number of elements.

Methods in which morphing wings are implemented are based on changing the shape of the wing during its operation, to maximize the performance or achieve other goals, such as noise reduction and include variation of chord length, swept angle, wingspan and angle of attack, as well as bending spanwise or chordwise. Morphing by varying the angle of attack is called twist morphing $[6,8,11]$.

Wing can be morphed in different ways, the simplest one was developed as a direct replacement for conventional mechanisms, promising improvements in parasitic drag by incorporating seamless skin, to reduce the parasitic drag on aerodynamic surfaces [9].

Table 1. Morphing solutions classification.

\begin{tabular}{|c|c|c|}
\hline Solution & Trailing Edge & Camber \\
\hline Shape Memory Alloy & SMA Actuation & SMA Actuated Skin \\
\hline Piezoelectric & Piezoelectric Actuation & $\begin{array}{l}\text { Piezoelectric Actuated } \\
\text { Skin }\end{array}$ \\
\hline
\end{tabular}

Muscle Function \& Plant-Movement

Nature Inspired

Hyper-Elliptic

Cambered Span [4]

\begin{tabular}{|c|c|c|c|}
\hline Torsional & & & Twist Morphing [11] \\
\hline Passive & & $\begin{array}{l}\text { Hexachiral Prismatic } \\
\text { Wingbox }\end{array}$ & \\
\hline \multirow{2}{*}{ Adaptive Compliant } & $\begin{array}{l}\text { Adaptive Compliant } \\
\text { Trailing Edge }\end{array}$ & $\begin{array}{l}\text { Integrated Compliant } \\
\text { Ribs }\end{array}$ & \\
\hline & $\begin{array}{l}\text { Mission Adaptive } \\
\text { Compliant Wing }\end{array}$ & & \\
\hline $\begin{array}{l}\text { Modified Classical } \\
\text { Mechanisms }\end{array}$ & Seamless Skin Flap & & \\
\hline
\end{tabular}

One simple method for changing the camber is the finger-like concept, where the rib is divided into segments connected by hinges in order to allow for the desired motion. 
Approaches based on rigid motion can also be found, such as piezoelectric actuators to excite a tab that moves a free-floating trailing edge flap, or SMA wire to rotate a tab for thickening the trailing edge [7].

Active skin made of an array of piezoceramic actuators can be used to achieve a deformation of the trailing edge [7].

Chiral honeycomb could be used as the internal structure of the aft section of a passive morphing (decambering) airfoil [5].

Compliant trailing edge section can be made, which is actuated with a SMA wire. The trailing edge is free to slide into the spar, thus allowing for large displacements. Similarly, the Mission Adaptive Compliant Wing (MACW) from FlexSys Inc. uses a compliant mechanism for morphing the trailing edge [2, 3].

Morphing trailing edge actuated by dielectric elastomers integrated in the skin was suggested. The rear part of the profile acts as a servo tab, thus reducing the required actuation forces [7].

Typical approach to morphing wing design is interdisciplinary, as shown on Figure 3. The challenge of achieving a transdisciplinary information exchange lies in the broad range of needed methods lying within those disciplines to manage [10].

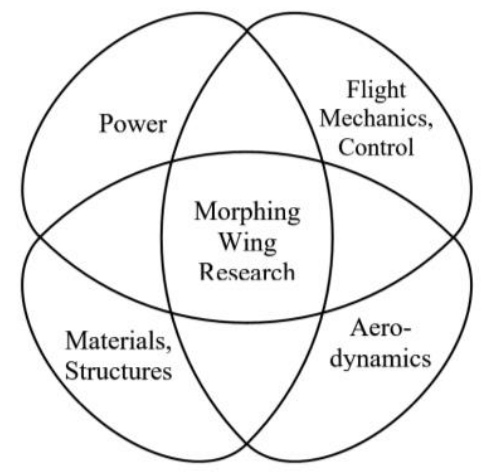

Figure 1. Typical Interdisciplinary approach.

\section{HALE aircraft}

High-Altitude Long Endurance (HALE) is the description of an air-borne vehicle which functions optimally at high-altitude (as high as $20 \mathrm{~km}$ ) and is capable of flights which last for considerable periods of time without recourse to landing.

Oracover foil as skin is widely used in HALE aircrafts as its leightweight. Plywood ribs can also be

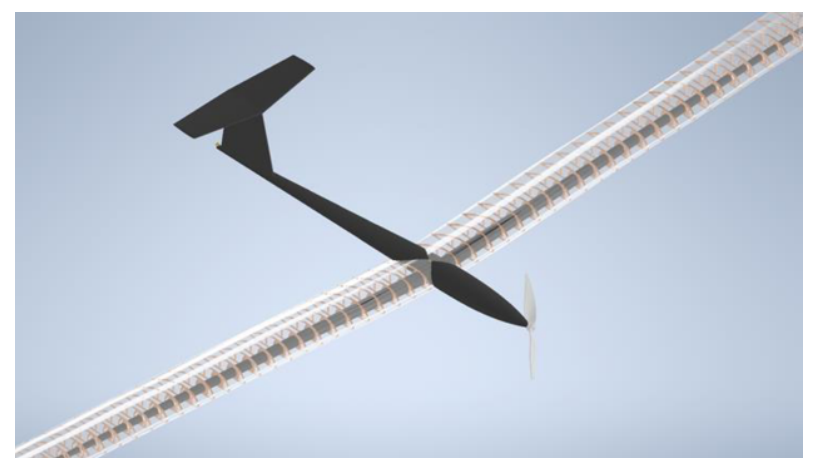

Figure 2. HALE aircraft. used. There is no need for using lift devices, as the take-off is performed from hand or 
using a small catapult device. Ailerons, elevator and rudder are used. Ribs usually have changing geometry along the wing. To reduce rib weight in the furthest ribs from the root, some additional cut outs can be put into place. An example of HALE aircraft is shown in Figures $2-3$.

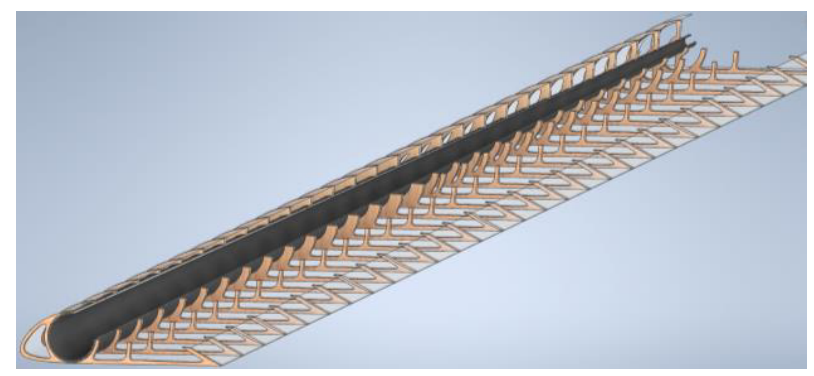

Figure 3. Wing structure, three-quarter section.

\section{Morphing wing concept}

The challenge morphing wing transdisciplinary design aims to solve is effective and efficient design development of said wing arrangements. Increased availability and introduction rate of efficient morphing wing designs for production are main expected impacts such methodology aims to achieve.

As the solution spans inventive methodology, aerodynamics, structures and materials, flight mechanics and control, the development process must be structured and as such comprises of four phases: inventive methodology approach utilisation to determine the design arrangement, flight mechanics and control considerations, structural and material considerations and aerodynamic verification. In the development process there should be an aircraft designer and preferably aerodynamics specialist, as well as mechanical engineer involved. The validation is up to the aircraft designer and should be comprised of aerodynamic analysis verification as well as direct comparison of arrangements.

To identify the best viable solution for the HALE aircraft, TRIZ methodology was utilized. TRIZ (Theory of the Resolution of Invention-Related Tasks) is a problemsolving, analysis and forecasting tool derived from the study of patterns of invention in the global patent literature the theory of inventive problem solving and occasionally goes by the English acronym TIPS.

This theory defines generalizable patterns in the nature of inventive solutions and the distinguishing characteristics of the problems that these inventions have overcome. An important part of the theory is revealing patterns of evolution and one of the objectives of TRIZ has been the development of an algorithmic approach to the invention of new systems, and to the refinement of existing ones. [12]

It is important to determine which features of the design ought to be improved. The idea of morphic wing is to improve adaptability, that's why it was chosen for the analysis as the main improved feature. Based on TRIZ decision matrix, the following principles would be used to determine the new design:

1. The principle of division:

1.1. Divide an object into independent parts

1.2. Make a disassembling object

1.3. Increase segmentation

2. The principle of versatility:

2.1. Make an object capable of carrying out several steps to replace the activities carried out by other parts of the object 
3. The principle of counterweight:

3.1. Balance the weight of an object by connecting to one or more other objects with lifting force

3.2. Balance the weight of the object by ensuring interaction with the environment (using aerodynamic forces)

4. The principle of dynamism:

4.1. Allow matching the characteristics of the object so as to make the operation optimal, or to move under the best operating conditions

4.2. Divide an object into elements that can move together

4.3. Make a flexible or adaptable object (or process) which was previously rigid and not flexible

5. The principle of local quality:

5.1. Go from the structure of a homogeneous object to a heterogeneous structure

5.2. Make each part of the object perform different activities in the best possible conditions

5.3. Specialize different parts of the object (make each part perform a different useful activity)

6. The principle of isolation:

6.1. Use flexible shells and thin films instead of three-dimensional structures

6.2. Isolate the object from the external environment using flexible shells and thin films

7. Inversion principle:

7.1. Reverse the useful action normally used to solve the problem

7.2. Immobilize mutually movable parts but set in motion parts that were still [1, 12-14].

The design that allows to achieve all of the above principles, which is an adaptive compliant structure proposed to meet these requirements, as well as its functioning model is shown in Figures $4-5$.

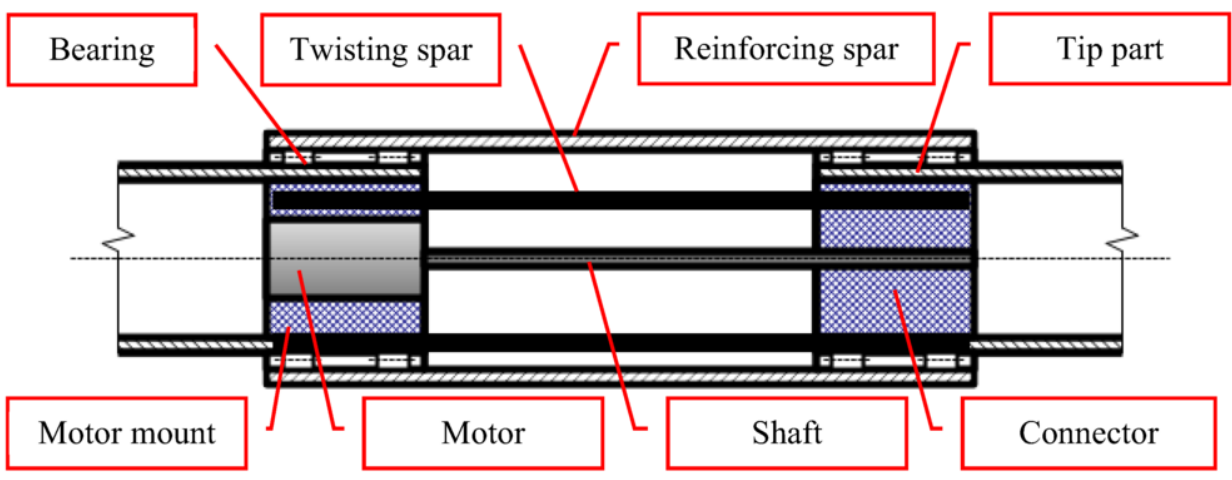

Figure 4. Wingtip Twist Morphing concept.

This design focuses on changing of the angle of attack of part of the wing at its tip. Spar is divided into 3 (unreinforced spar) or 4 (reinforced spar) elements. The tip-most part is allowed to twist by incorporating a split tubular spar, incorporated in and supported by an outside tubular spar (in reinforced spar version), that is put into place solely to carry the bending loads. 
Twist of the wing is controlled by servomotor fixed to the spar. Torque generated by the servomotor is carried through an elastic clutch by a CFRP shaft to the second fixing point.

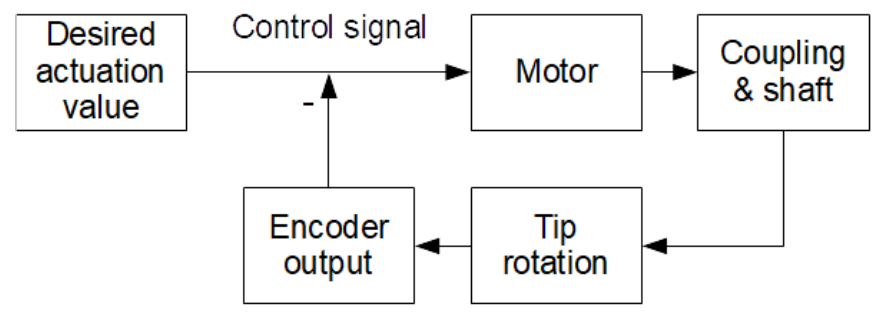

Figure 5. Wingtip Twist Morphing working principle.

\section{Analysis}

The analysis was performed on HALE aircraft under development in SkyTech eLab „Twin Stratos”.

During the analysis the main problem that should be solved is the aerodynamic performance of considered design. The airplane structure was reconstructed in XFLR5 software as shown in Figure 6.

The aircraft main wing is divided into several sections with differing chord and dihedrals. Different versions of roll control elements are taken into account. The following analysis compares 3 options of control - clasical with ailreons, tail control, wingtip twist morphing.

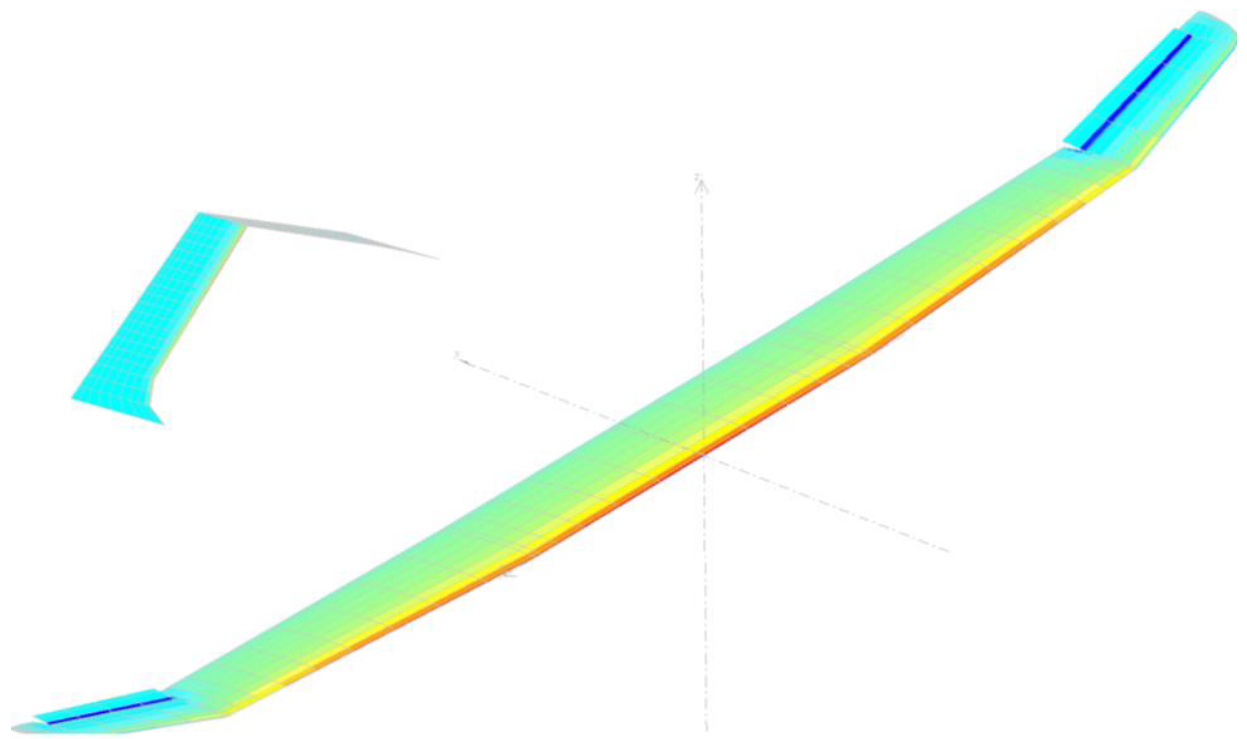

Figure 6. Considered aircraft reconstructed in XFLR5 software.

Data regarding lift coefficient was gathered and processed for the purpose of calculations and rendered results shown in Figures below. Figures $7-9$ show lift 
coefficient distributions in all considered arrangements, for $0^{\circ}, 5,5^{\circ}$ and $10^{\circ}$ angle of attack.

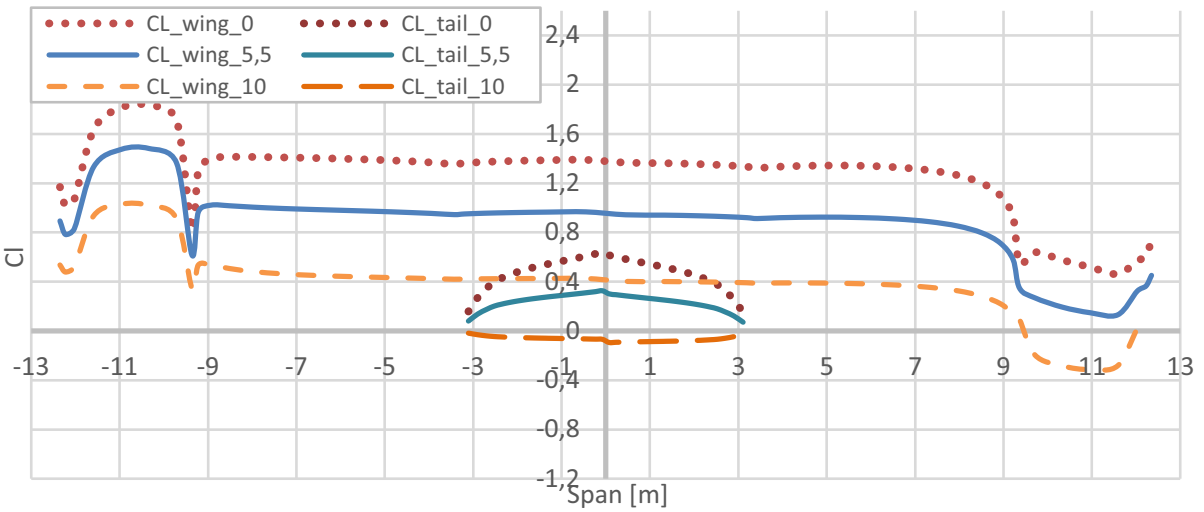

Figure 7. Lift coefficient distribution (ailerons).

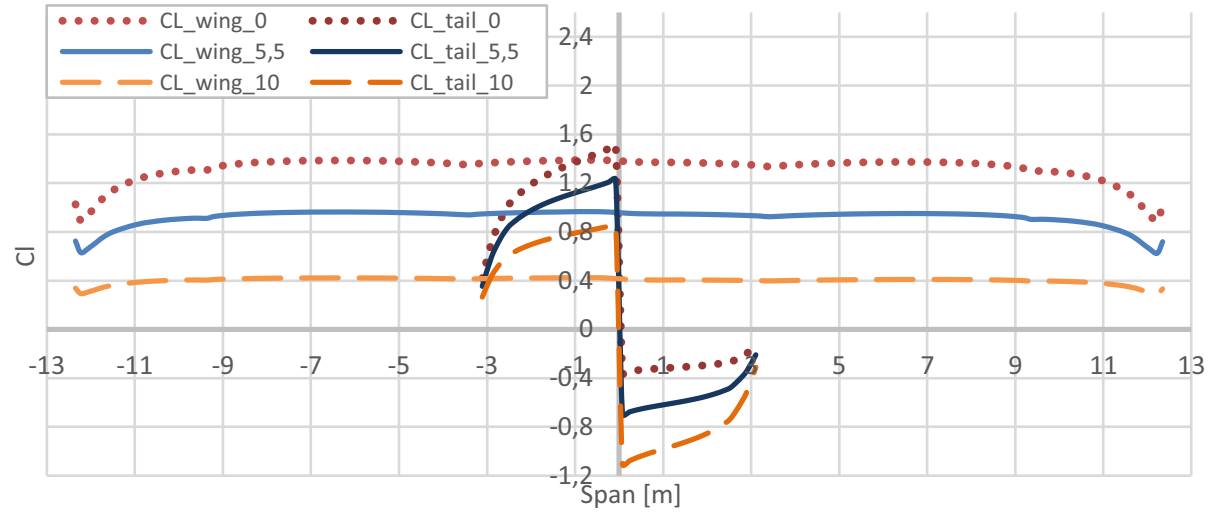

Figure 8. Lift coefficient distribution (tail compliant trailing edge).

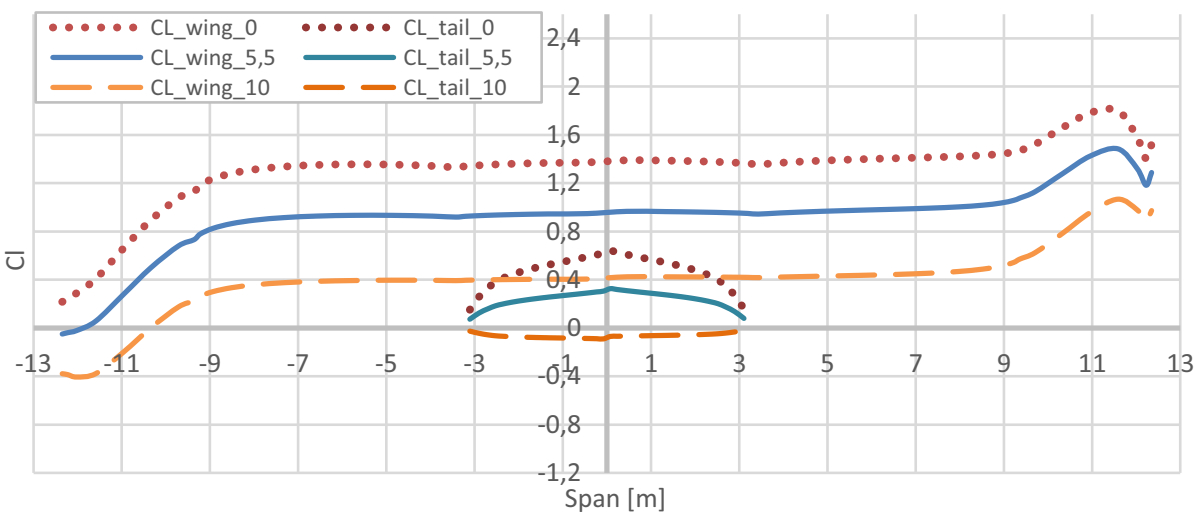

Figure 9. Lift coefficient distribution (wingtip twist morphing). 
To conclude the analysis, the data was gathered to calculate both lift and rolling moment for all considered arrangements. The total lift and rolling moment, as well as their respective improvements in comparison to the classical control system are shown in Tables $2-3$.

Lift increased significantly in the tail control arrangement and reached about 5,57\% increase for $0^{\circ}$ angle of attack. Results for Twist Morphing show, that the improvement is much less dependent on AoA.

Table 2. Lift and its improvement in comparison to classical control system.

\begin{tabular}{ccccc}
\hline AoA [deg] & Aileron control & Tail control & Twist Morphing (wing tip) & \\
\hline 0 & 672 & 710 & 707 & L [N] \\
& 0.00 & 5.57 & 5.14 & Impr. [\%] \\
5.5 & 1662 & 1751 & 1743 & L [N] \\
& 0.00 & 5.38 & 4.88 & Impr.[\%] \\
10 & 2437 & 2567 & 2554 & L [N] \\
& 0.00 & 5.34 & 4.82 & Impr. [\%] \\
\hline
\end{tabular}

Rolling moment increased in both arrangements and reached about $9 \%$ increase for $0^{\circ}$ angle of attack in the tail control arrangement. Twist Morphing yielded an increase of over $6 \%$.

Table 3. Rolling moment and its improvement in comparison to classical control system.

\begin{tabular}{ccccc}
\hline AoA [deg] & Aileron control & Tail control & Twist Morphing (wing tip) & \\
\hline 0 & 4670 & 5091 & 4984 & $\mathrm{M} \mathrm{[Nm]}$ \\
& 0.00 & 9.00 & 6.72 & Impr. [\%] \\
5.5 & 10725 & 11662 & 11473 & $\mathrm{M} \mathrm{[Nm]}$ \\
10 & 0.00 & 8.74 & 6.97 & Impr. [\%] \\
& 15458 & 16799 & 16545 & $\mathrm{M}[\mathrm{Nm}]$ \\
\hline
\end{tabular}

Figures $10-13$ shown below give a representation of results of lift and rolling moment. Improvements are presented in Figures $12-13$. 


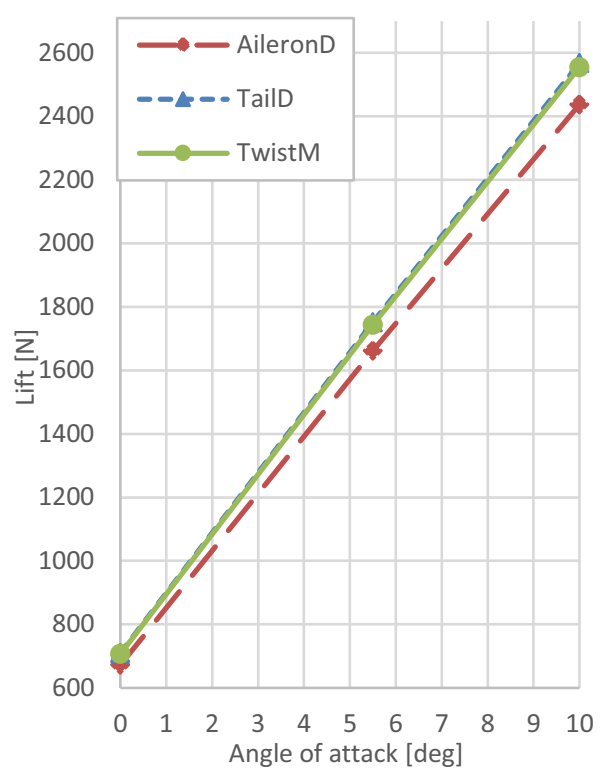

Figure 10. Lift vs angle of attack.

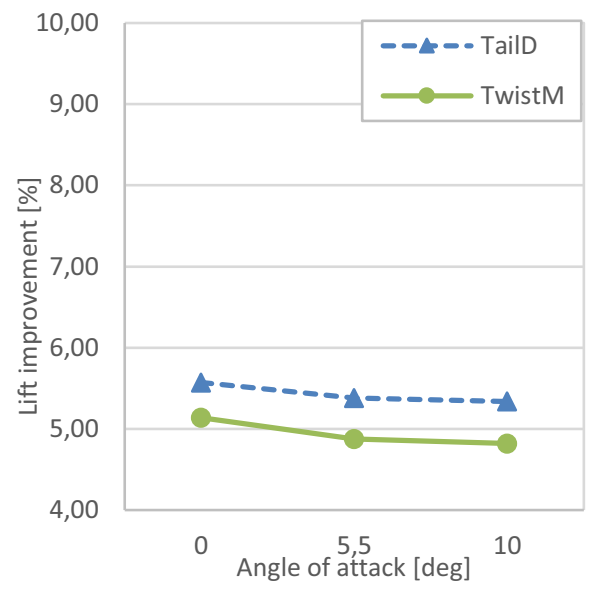

Figure 12. Lift improvement vs AoA.

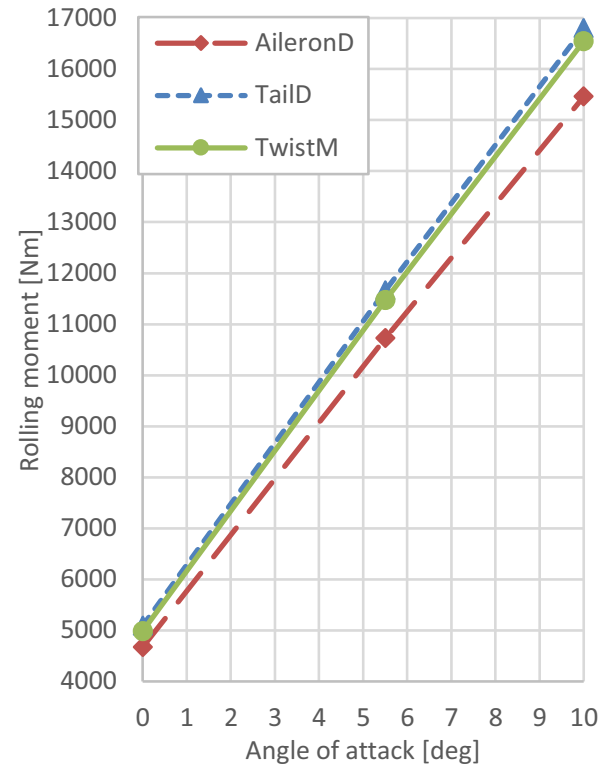

Figure 11. Rolling moment vs angle of attack.

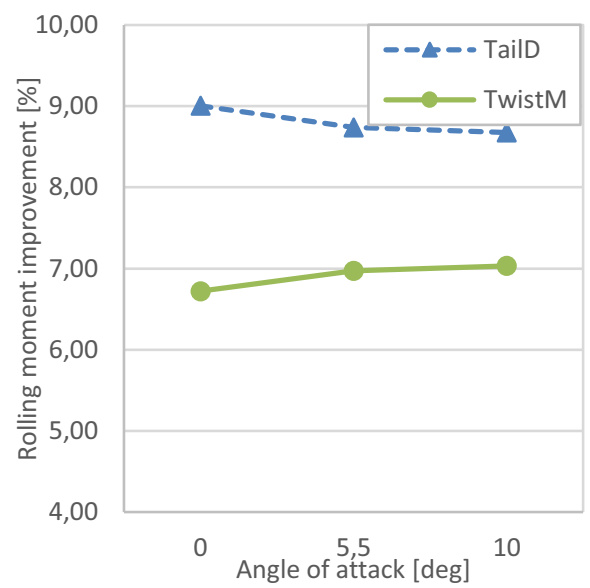

Figure 13. Rolling moment improvement vs AoA.

\section{Conclusions}

The considered arrangements of control surfaces were able to improve the rolling moment in all considered angle of attack scenarios by several percent. Additionally, lift also increased in comparison to classical control system, which in addition to reduced parasitic drag concludes, that such arrangements can be desirable for utilisation in High Altitude Long Endurance UAVs. For the considered aircraft and angle of attack ranges, the tail control arrangement offers the best improvements of up to $9 \%$. 


\section{Acknowledgement}

We wanted to express gratitude for SkyTech eLab company for providing technical documentation of the Twin Stratos UAV project for research purposes.

\section{References}

[1] G. Altshuller, A. Seredinski, 40 Principes d'innovation TRIZ pour toutes applications, Seredinski, Paris, 2004

[2] C. Y. Herrera, N. D. Spivey, S. Lung, G. Ervin, P. Flick, Aeroelastic Airworthiness Assessment of the Adaptive Compliant Trailing Edge Flaps, 46th Society of Flight Test Engineers International Symposium, Lancaster, 2015, https://ntrs.nasa.gov/archive/nasa/casi.ntrs.nasa.gov/20150020900.pdf, accessed July,1 2020.

[3] S. Kota, R. Osborn, G. Ervin, D. Maric, P. Flick, D. Paul, Mission Adaptive Compliant Wing - Design, Fabrication and Flight Test, RTO-MP-AVT-168, 2009

[4] J. Manzo, E. Garcia, A. Wickenheisera, G. C. Horner, Adaptive structural systems and compliant skin technology of morphing aircraft structures, Smart Structures and Materials 2004: Smart Structures and Integrated Systems, Vol. 5390, 2004, https://doi.org/10.1117/12.540348.

[5] J. Martin, J. J. Heyder-Bruckner, C. Remillat, F. Scarpa, K. Potter, M. Ruzzene: The hexachiral prismatic wingbox concept, Physica Status Solidi (b) , 2008, Vol. 245, No. 3, 570-577.

[6] G. Molinari, A. F. Arrieta, P. Ermanni, Aero-Structural Optimization of Three-Dimensional Adaptive Wings with Embedded Smart Actuators, American Institute of Aeronautics and Astronautics Journal, Vol. 52, No. 9, 2014, https://doi.org/10.2514/1.J052715.

[7] F. Previtali, P. Ermanni, Performance of a non-tapered 3D morphing wing with integrated compliant ribs, Smart Materials and Structures 21, 2012, DOI: 10.1088/0964-1726/21/5/055008.

[8] V. Prisacariu, M. Boscoianu, I. Cîrciu, Morphing wing concept for small UAV, Applied Mechanics and Materials, Vol. 332, 2013, pp. 44-49.

[9] G. A. A. Thuwis, M. M. Abdalla, Z. Gürdal, Optimization of a variable-stiffness skin for morphing highlift devices, Smart Materials and Structures, Vol. 19, 2010, pp. 1-10.

[10] S. Vasista, L. Tong, K.C. Wong, Realization of Morphing Wings: A Multidisciplinary Challenge, Journal of Aircraft, Vol. 49, No. 1, 2012, https://doi.org/10.2514/1.C031060.

[11] R. Vos, Z. Gürdal, M. Abdalla, Mechanism for Warp-Controlled Twist of a Morphing Wing, Journal of Aircraft, Vol. 47, No. 2, March-April 2010, https://doi.org/10.2514/1.39328.

[12] https://en.wikipedia.org/wiki/TRIZ

[13] Oxford Creativity, Accessed: 10.02.2020. [Online]. Available: https://www.triz.co.uk/

[14] TRIZ40 V2.1, Accessed: 10.02.2020. [Online]. Available: http://www.triz40.com/TRIZ_GB.php 